\title{
A Quantifier Elimination Algorithm for Linear Modular Equations and Disequations *
}

\author{
Ajith K John ${ }^{1}$ and Supratik Chakraborty ${ }^{2}$ \\ 1 Homi Bhabha National Institute, BARC, Mumbai, India \\ 2 CFDVS, Dept. of Computer Sc. \& Engg., IIT Bombay, India
}

\begin{abstract}
We present a layered bit-blasting-free algorithm for existentially quantifying variables from conjunctions of linear modular (bitvector) equations (LMEs) and disequations (LMDs). We then extend our algorithm to work with arbitrary Boolean combinations of LMEs and LMDs using two approaches - one based on decision diagrams and the other based on SMT solving. Our experiments establish conclusively that our technique significantly outperforms alternative techniques for eliminating quantifiers from systems of LMEs and LMDs in practice.
\end{abstract}

\section{Introduction}

Quantifier elimination (QE) is the process of converting a formula containing existential and/or universal quantifiers in a suitable logic into a semantically equivalent quantifier-free formula. Formally, let $A$ be a quantifier-free formula over a set $X$ of free variables in a first-order theory $\mathcal{T}$. Consider the quantified formula $Q_{1} y_{1} Q_{2} y_{2} \ldots Q_{m} y_{m}$. A, where $Y=\left\{y_{1}, \ldots y_{m}\right\}$ is a subset of $X$, and $Q_{i} \in\{\exists, \forall\}$ for $i \in\{1, \ldots m\}$. QE computes a quantifier-free formula $A^{\prime}$ with free variables in $X \backslash Y$ such that $A^{\prime} \equiv_{\mathcal{T}} Q_{1} y_{1} Q_{2} y_{2} \ldots Q_{m} y_{m} . A$, where $\equiv_{\mathcal{T}}$ denotes semantic equivalence in theory $\mathcal{T}$. This has a number of important applications in formal verification and program analysis. Example applications include computing abstractions of symbolic transition relations, computing strongest postconditions of program statements and computing interpolants in CEGAR frameworks. Since $\forall y . \varphi \equiv \neg \exists y . \neg \varphi$ in all first-order theories, it suffices to focus on algorithms for eliminating existential quantifiers. This paper presents one such algorithm for a fragment of the theory of bit-vectors that we have found useful in verification of word-level RTL designs.

Currently, the most popular technique for eliminating quantifiers from bitvector formulae involves blasting bit-vectors into individual bits (Boolean variables), followed by quantification of the blasted Boolean variables. This approach has some undesirable features. For example, blasting involves a bitwidthdependent blow-up in the size of the problem. This can present scaling problems in the usage of Boolean reasoning tools (e.g. BDD based tools), especially when reasoning about wide words. Similarly, given an instance of the QE problem, blasting variables that are quantified may transitively require blasting other variables (that are not quantified) as well. This can cause the quantifier-eliminated

\footnotetext{
* This work was supported by a research grant from Board of Research in Nuclear
} Sciences, India. 
formula to appear more like a propositional formula on blasted bits, instead of being a bit-vector formula. Since reasoning at the level of bit-vectors is often more efficient in practice than reasoning at the level of blasted bits, QE using bit-blasting may not be the best option if the quantifier-eliminated formula is intended to be used in further bit-vector level reasoning. This motivates us to ask if we can efficiently eliminate quantifiers in the theory of bit-vectors without resorting to bit-blasting (or model enumeration) in practice. Ideally, we would have liked to obtain such a QE procedure for the entire theory of bit-vectors. Unfortunately, we do not have this yet. We therefore focus on a fragment of the theory, namely Boolean combinations of equations and disequations of bitvectors, that we have found useful in word-level verification of RTL designs, and present a QE procedure for this fragment.

Since bit-vector arithmetic is the same as modular arithmetic on integers, our algorithm can also be viewed as one for existentially quantifying variables from a Boolean combination of linear modular integer equations and disequations.

A Linear Modular Equation (LME) is an equation of the form $c_{1} \cdot x_{1}+\cdots+$ $c_{n} \cdot x_{n}=c_{0} \quad\left(\bmod 2^{p}\right)$ where $p$ is a positive integer constant, $x_{1}, \ldots, x_{n}$ are $p$-bit non-negative integer variables, and $c_{0}, \ldots, c_{n}$ are integer constants in $\left\{1, \ldots, 2^{p}-\right.$ 1\}. Similarly, a Linear Modular Disequation (LMD) is a disequation of the form $c_{1} \cdot x_{1}+\cdots+c_{n} \cdot x_{n} \neq c_{0} \quad\left(\bmod 2^{p}\right)$. Conventionally, $2^{p}$ is called the modulus of the LME or LMD. For notational convenience, we will henceforth use "LMC" to refer to an LME or LMD. Since every variable in an LMC $c_{1} \cdot x_{1}+\cdots+c_{n} \cdot x_{n} \bowtie c_{0}$ $\left(\bmod 2^{p}\right)$, where $\bowtie \in\{=, \neq\}$, represents a $p$-bit integer, it follows that a set of LMCs sharing a variable must have the same modulus. However, there are applications where we need to consider Boolean combinations of LMCs that do not share any variable, and have different moduli. In such cases, we propose to appropriately shift the moduli of LMCs, so that all LMCs have the same modulus. This can always be done since the LMCs $\lambda_{1} \equiv c_{1} \cdot x_{1}+\cdots+c_{n} \cdot x_{n} \bowtie c_{0}$ $\left(\bmod 2^{p}\right)$ and $\lambda_{2} \equiv 2^{q} \cdot c_{1} \cdot x_{1}^{\prime}+\cdots+2^{q} \cdot c_{n} \cdot x_{n}^{\prime} \bowtie 2^{q} \cdot c_{0} \quad\left(\bmod 2^{p+q}\right)$ are related in the following way: every solution of $\lambda_{1}$ can be bit-extended to give a solution for $\lambda_{2}$, and every solution of $\lambda_{2}$ can be bit-truncated to give a solution for $\lambda_{1}$. Hence, using $\lambda_{2}$ in place of $\lambda_{1}$ suffices for checking satisfiability and also for finding solutions of Boolean combinations of LMCs. In the remainder of this paper, we will assume without loss of generality that whenever we consider a set of LMCs, all of them have the same modulus.

Our primary motivation comes from bounded model checking (BMC) of word-level RTL designs. As an example, consider the synchronous circuit shown in Fig. 1, with the relevant part of its functionality described in VHDL with the figure. The thick shaded arrows and the thin solid arrows in the figure represent 8-bit words and 1-bit lines respectively.

The circuit comprises a controller and two 8-bit registers, $A$ and $B$. The controller switches between two states, 0 and 1, depending on the value of $A$. In state $0, A$ works as a down-counter until it reaches $0 \times 00^{3}$, in which case $A$ loads itself with an input value from InA and the controller switches to state

\footnotetext{
${ }^{3}$ We use the $0 x$ prefix to denote hexadecimal values.
} 


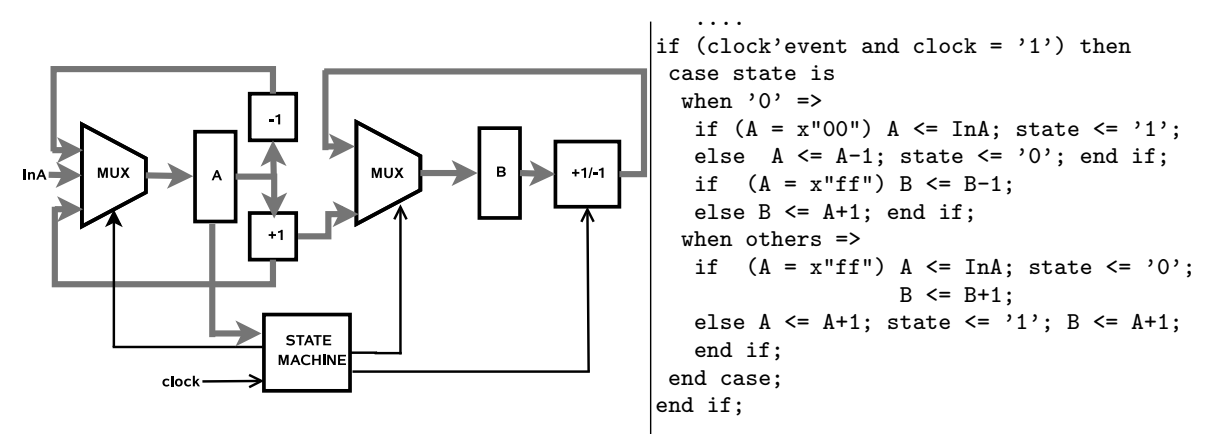

Fig. 1. An Example Circuit

1. In state $1, A$ works as an up-counter until it reaches $0 x f f$, in which case it loads the value from $\operatorname{In} A$ and the controller switches to state 0 . Register $B$ is always loaded with the value of $A+1$ except when $A$ has the value $0 x f f$. If this happens in state 0 (down-counting state), $B$ decrements its previously stored value; otherwise, $B$ increments its previously stored value.

A word-level transition relation, $R$, for this circuit can be obtained by conjoining the following three equality relations, where all operations on $A$ and $B$ are assumed to be modulo $2^{8}$.

$$
\begin{aligned}
\text { state }^{\prime} & =\text { ite }(\text { state }=0, \text { ite }(A=0 \times 00,1,0), \text { ite }(A=0 x f f, 0,1)) \\
A^{\prime} & =\text { ite }(\text { state }=0, \text { ite }(A=0 \times 00, \ln A, A-1), \text { ite }(A=0 x f, \ln A, A+1)) \\
B^{\prime} & =\text { ite }(\text { state }=0, \text { ite }(A=0 x f f, B-1, A+1), \text { ite }(A=0 x f f, B+1, A+1))
\end{aligned}
$$

In the above relations, state ${ }^{\prime}, A^{\prime}$ and $B^{\prime}$ refer to values of state, $A$ and $B$ after the next rising edge of the clock. Note also that $A, A^{\prime}, B$ and $B^{\prime}$ are 8 -bit wide bit-vector variables and state and state ${ }^{\prime}$ are propositional variables. Since $R$ is a conjunction of equalities involving ite, and since $a=i t e(b, c, d)$ represents $(b \wedge(a=c)) \vee(\neg b \wedge(a=d)), R$ is essentially a Boolean combination of LMCs.

The above circuit has the property that once started in state 0 , it never reaches state 1 with $0 \times 00$ in register $B$. Suppose we wish to use BMC to prove that this property holds for the first $N$ cycles of operation. This can be done by unrolling the transition relation $N$ times, conjoining the unrolled relation with the negation of the property, and then checking for satisfiability of the resulting constraint using an SMT solver that can reason about bit-vectors. Since $R$ contains all variables (in unprimed and primed versions) that appear in the RTL description, unrolling $R$ a large number of times gives a constraint with a large number of variables. This problem is particularly acute for circuits with a large number of internal state variables. While the number of variables in a constraint is not the only factor that affects the performance of an SMT solver, for large enough values of $N$, the increased variable count indeed has an adverse effect on the performance of the solver, as indicated by our experiments.

In order to alleviate the above problem, one can use an abstract transition relation $R^{\prime}$ that relates only a chosen subset of variables relevant to the property being checked, while abstracting the relation between the other variables. In our example, we can compute such an $R^{\prime}$ by existentially quantifying the bit-vector variables $\mathrm{A}$ and $\mathrm{A}^{\prime}$ from $R$. This gives $R^{\prime}$ as:

$$
\left(\left(\text { state }^{\prime}=1\right) \wedge\left(\mathrm{B}^{\prime}=0 \times 01\right)\right) \vee
$$




$$
\begin{gathered}
\left(\left(\text { state }^{\prime}=0\right) \wedge\left(\mathrm{B}^{\prime}=\text { ite }(\text { state }=0, \mathrm{~B}-1, \mathrm{~B}+1)\right)\right) \\
\left(\left(\text { state }^{\prime}=\text { state }\right) \wedge\left(\mathrm{B}^{\prime} \neq 0 \times 00\right) \wedge\left(\mathrm{B}^{\prime} \neq 0 \times 01\right)\right)
\end{gathered}
$$

On careful examination, it can be seen that if we unroll $R^{\prime}$ (instead of $R$ ) during BMC, we can still prove that the circuit never reaches state 1 with $\mathrm{B}$ set to $0 \times 00$ if it starts in state 0 . Since $R^{\prime}$ contains fewer variables than $R$, the constraint obtained by unrolling $R^{\prime}$ has fewer variables. In general, this can lead to significantly better performance of the back-end SMT solver, as demonstrated in our experiments.

The example presented above is representative of a more general scenario. In general, Boolean combinations of LMCs arise when building transition relations for RTL designs and/or embedded systems containing conditional statements that check for equalities of words/registers. Building an abstract transition relation in such cases requires existentially quantifying variables from Boolean combinations of LMCs. Obtaining the abstract transition relation at the wordlevel is particularly appealing since it allows word-level reasoning to be applied to the abstraction. This motivates us to study the problem of eliminating quantifiers from Boolean combinations of LMCs without resorting to bit-blasting (or model enumeration) in practice.

Contributions. There are two primary contributions of this paper. First, we describe a bit-blasting-free algorithm for eliminating quantifiers from conjunctions of LMCs. The algorithm is based on a layered approach, i.e., the cheaper layers are invoked first and more expensive layers are called only when required. Later, we extend this to QE algorithm for Boolean combinations of LMCs. While our algorithm uses a final layer of model enumeration for the sake of theoretical completeness, extensive experiments indicate that we never need to invoke this layer in practice. Our second contribution is an extensive set of carefully conducted experiments that not only demonstrate the effectiveness of our approach over alternative techniques, but also allows us to identify criteria for choosing the right QE technique for a given problem instance.

Related Work. Several interesting approaches have been proposed earlier for reasoning about LMEs (e.g., [6,7]). Although our study indicates that nontrivial counts of LMDs appear in constraints arising from real verification problems, LMDs have traditionally received relatively less attention. Jain et al [7] showed that the satisfiability problem for a conjunction of LMCs is NP-hard. However, their work subsequently focused on systems of LMEs and Linear Diophantine Equations and Disequations, and discussed algorithms to compute interpolants in such systems. Bit-blasting [3] followed by bit-level QE is arguably the dominant technique used in practice for eliminating quantifiers from bitvector constraints. As discussed earlier, this approach, though simple, destroys the word-level structure of the problem and does not scale well for LMCs with large modulus. Since LMEs and LMDs can be expressed as formulae in Presburger Arithmetic (PA) [3], QE techniques for PA (e.g.those in [5]) can also be used to eliminate quantifiers from Boolean combinations of LMCs. Similarly, automata-theoretic approaches for eliminating quantifiers from PA formulae [8] can also be used. However once converted to PA formulae, converting back to 
Boolean combinations of LMCs is difficult. Also, empirical studies have shown that using PA techniques to eliminate quantifiers from Boolean combinations of LMCs often blows up in practice [3]. The work that is most closely related to our is that of Ganesh and Dill [6]. The authors of [6] present a technique for reducing LMEs to a solved form by selecting variables in a specific order. While this does not directly give us a technique to eliminate a user-specified variable from a conjunction of LMEs, their work can be extended to achieve this. More importantly, [6] does not consider the problem of eliminating variables in constraints involving LMDs. This problem is addressed in our current work.

\section{Quantifier Elimination for a Conjunction of LMCs}

The problem we wish to solve in this section can be formally stated as follows. Given a set of LMCs over variables $x_{1}, \ldots, x_{n}$, let $A$ denote the conjunction of the LMCs. Without loss of generality, we wish to compute $A^{\prime} \equiv \exists x_{1} \cdots \exists x_{t}$. $A$, where $A^{\prime}$ is a Boolean combination of LMCs. For reasons of succinctness, we also require that $A^{\prime}$ contains no ground terms other than integer constants, and no ground (sub-)formulas other than true and false. This problem is easily seen to be NP-hard. This follows from the facts: (i) the satisfiability problem for a conjunction of LMCs is NP-hard, even when all moduli are a priori fixed to 4 (see [7]), and (ii) a conjunction of LMCs $A$ over $x_{1}, \ldots, x_{n}$ is satisfiable iff an algorithm for computing $A^{\prime} \equiv \exists x_{1} \cdots \exists x_{n}$. A returns true (due to the succinctness requirement of $A^{\prime}$ ).

Since an algorithm for computing $\exists x_{i}$. $A$ can be used in an iterative way to compute $\exists x_{1} \cdots \exists x_{t}$. A, we will initially focus on the (seemingly simpler) problem of computing $\exists x_{i}$. $A$ in the subsequent discussion. All LMCs considered in the remainder of this section have modulus $2^{p}$, for some positive integer $p$, unless stated otherwise. For notational clarity, we will therefore omit mentioning " $\left(\bmod 2^{p}\right) "$ with LMCs in the following discussion. We have skipped the proofs of lemmas and the details of some procedures which can be found in a detailed version of this paper [13].

In the following discussion, we use names starting with " $Q E 1$ " and " $Q E$ " for procedures to eliminate a single quantifier and multiple quantifiers respectively.

Lemma 1. An $L M C c_{1} \cdot x_{1}+\cdots+c_{n} \cdot x_{n} \bowtie c_{0}$ can be equivalently expressed as $2^{k_{1}} \cdot x_{1} \bowtie t_{1}$, where $\bowtie \in\{=, \neq\}, t_{1}$ is a term free of $x_{1}$ and $k_{1}$ is an integer such that $0 \leq k_{1} \leq p-1$.

Example: All LMCs in this example have modulus 8. Consider the LME $6 x+$ $4 y=0$. Rearranging the terms modulo 8 , we get $3 \cdot 2^{1} x=4 y$. Multiplying by 3 (multiplicative inverse of 3 modulo 8 ) and simplifying gives, $2^{1} x=4 y$.

For brevity, henceforth whenever we express LMCs as $2^{k_{i}} \cdot x_{1} \bowtie t_{i}$ where $\bowtie \in\{=, \neq\}$, we will omit mentioning " $t_{i}$ is a term free of $x_{1}$ and $k_{i}$ is an integer such that $0 \leq k_{i} \leq p-1$ ".

Lemma 2. $\exists x_{1} \cdot\left(2^{k_{1}} \cdot x_{1}=t_{1}\right) \equiv\left(2^{p-k_{1}} \cdot t_{1}=0\right)$

Example: All LMCs in this example have modulus 8. $\exists y \cdot\left(2^{1} \cdot y=5 \cdot x+2\right) \equiv$ $\left(2^{3-1} \cdot(5 \cdot x+2)=0\right) \equiv(4 \cdot x=0)$ 
Lemma 3. Let $A$ be the conjunction of $m$ LMEs of the form $2^{k_{i}} \cdot x_{1}=t_{i}$, where $i$ ranges from 1 through $m$. Then $\exists x_{1}$. A can be equivalently expressed as a conjunction of LMEs each of which is free of $x_{1}$.

Example: All LMCs in this example have modulus 8. Consider the problem of computing $\exists y .\left(\left(2^{1} y=5 x+2\right) \wedge\left(2^{2} y=5 x+6 z\right) \wedge\left(2^{1} y=2 x+4\right)\right)$. This can be equivalently expressed as $\exists y \cdot((2 y=5 x+2) \wedge(2 \cdot(5 x+2)=5 x+6 z)$ $\wedge(5 x+2=2 x+4))$. Simplifying modulo 8 , we get $\exists y \cdot((2 y=5 x+2)) \wedge(5 x+$ $2 z=4) \wedge(3 x=2)$. Using Lemma 2 , we obtain the final result as $(4 x=0)$ $\wedge(5 x+2 z=4) \wedge(3 x=2)$.

Lemma 4. Let $A$ be the conjunction of $r$ LMCs of the form $2^{k_{i}} \cdot x_{1} \bowtie t_{i}$, where $\bowtie \in\{=, \neq\}$ and $i$ ranges from 1 through $r$. Let $2^{k_{1}} \cdot x_{1}=t_{1}$ be the LME with the minimum $k_{i}$ among all LMEs in $A$. Then $\exists x_{1} . A \equiv \psi_{1} \wedge \exists x_{1} . \psi_{2}$, where $\psi_{1}$ is a conjunction of LMCs independent of $x_{1}$, and $\psi_{2}$ is a conjunction of LMCs with at most one LME $2^{k_{1}} \cdot x_{1}=t_{1}$. In addition, $\psi_{2}$ contains only those LMDs in $A$ in which the coefficient of $x_{1}$ is of the form $2^{k_{i}}$, where $k_{i}<k_{1}$.

Example: All LMCs in this example have modulus 8. Consider the problem of computing $\exists y \cdot\left(\left(2^{1} y=5 x+2\right) \wedge\left(2^{2} y=5 x+6 z\right) \wedge\left(2^{1} y \neq 2 x+4\right) \wedge\left(2^{0} y \neq\right.\right.$ $6 x+7 z))$. This can be equivalently expressed as $\exists y \cdot((2 y=5 x+2) \wedge(2 \cdot(5 x+2)=$ $5 x+6 z) \wedge(5 x+2 \neq 2 x+4) \wedge(y \neq 6 x+7 z))$. Simplifying modulo 8 , we get $(5 x+2 z=4) \wedge(3 x \neq 2) \wedge \exists y .((2 y=5 x+2) \wedge(y \neq 6 x+7 z))$. Note that $\psi_{1}$ and $\exists x_{1} . \psi_{2}$ here are $(5 x+2 z=4) \wedge(3 x \neq 2)$ and $\exists y \cdot((2 y=5 x+2) \wedge(y \neq 6 x+7 z))$ respectively.

The above results immediately yield two simple algorithms: (a) QE1_1LME that takes an LME and a variable to quantify out, and returns the equivalent quantifier-free formula (based on Lemma 2), and (b)QE1_Layer1 that takes a conjunction of LMCs and a variable $x_{1}$ to quantify out and returns the equivalent conjunction of $\psi_{1}$ and $\exists x_{1} . \psi_{2}$ (as given by Lemma 4 ).

If the $k_{i}$ 's of all LMDs in $A$ are such that $k_{1} \leq k_{i}$, then $\exists x_{1} \cdot \psi_{2}$ reduces to $\exists x_{1} \cdot\left(2^{k_{1}} \cdot x_{1}=t_{1}\right)$. According to Lemma 2 , this is equivalent to $2^{p-k_{1}}$. $t_{1}=0$. Hence, in this case, algorithms QE1_Layer1 and QE1_1LME suffice to compute $\exists x_{1}$. A. In general, however, $\exists x_{1} . \psi_{2}$ may contain LMDs containing $x_{1}$ that require further processing before $x_{1}$ is eliminated. We describe techniques for doing this in the following subsections.

\subsection{Dropping Unconstraining LMDs}

We now consider the problem of simplifying $\exists x_{1} . \psi_{2}$ obtained above, when $\exists x_{1} . \psi_{2}$ contains LMDs. Let $\psi_{2} \equiv \xi \wedge \lambda$, where $\lambda$ is an LMD and $\xi$ is a conjunction of LMCs. We say that $\lambda$ is unconstraining in $\exists x_{1} . \psi_{2}$ iff $\exists x_{1} .(\xi \wedge \lambda) \equiv \exists x_{1}$. $\xi$. Unconstraining LMDs can simply be dropped from $\exists x_{1} \cdot \psi_{2}$, thereby simplifying the task of QE. Unfortunately, identifying all unconstraining LMDs from $\psi_{2}$ involves invoking an SMT solver for quantified bit-vector formulas. In this subsection, we present a sound technique for identifying a subset of unconstraining LMDs in $\exists x_{1} \cdot \psi_{2}$. Our approach exploits the fact that an LMD is satisfied even if a single bit in the left-hand side of the LMD differs from the corresponding bit in the 
right-hand side. We therefore propose to identify LMDs in $\exists x_{1} \cdot \psi_{2}$ that can be satisfied by selectively assigning values to specific bits of $x_{1}$, without causing any other LME or LMD in $\exists x_{1} . \psi_{2}$ to be violated. Since $x_{1}$ is existentially quantified, these LMDs are effectively unconstraining in $\exists x_{1} \cdot \psi_{2}$. We illustrate this idea below through an example.

Consider the problem $\exists x .(\xi \wedge \lambda)$ where $\xi \equiv(4 x=6 y+2 z) \wedge(2 x \neq 2 y+4 z) \wedge$ $(2 x \neq 6 y+6 z)$ and $\lambda \equiv(x \neq y+z)$, and all LMCs have modulus 8. For clarity of exposition, we use the notation $x[i]$ to denote the $i^{\text {th }}$ bit of a bit-vector $x$, and adopt the convention that $x[0]$ denotes the least significant bit of $x$. We claim that any solution of $\xi$ can be "engineered" by possibly modifying the value of $x[2]$ to give a solution of $\xi \wedge \lambda$, and vice versa. In order to see why this is true, note that the LME $4 x=6 y+2 z$ constrains only $x[0]$ and the LMDs $(2 x \neq 2 y+4 z)$, $(2 x \neq 6 y+6 z)$ constrain only $x[0]$ and $x[1]$. Therefore, the value of $x[2]$ does not affect satisfaction of $\xi$. Any solution of $\xi$ can therefore be engineered to a solution of $\xi \wedge \lambda$ by ensuring that $x[2]$ differs from the most-significant bit of $y+z$. Hence, $\exists x .(\xi) \Rightarrow \exists x .(\xi \wedge \lambda)$. The converse, i.e. $\exists x .(\xi \wedge \lambda) \Rightarrow \exists x .(\xi)$ obviously holds. Hence in this example, $(x \neq y+z)$ is an unconstraining LMD in $\exists x .(\xi \wedge \lambda)$.

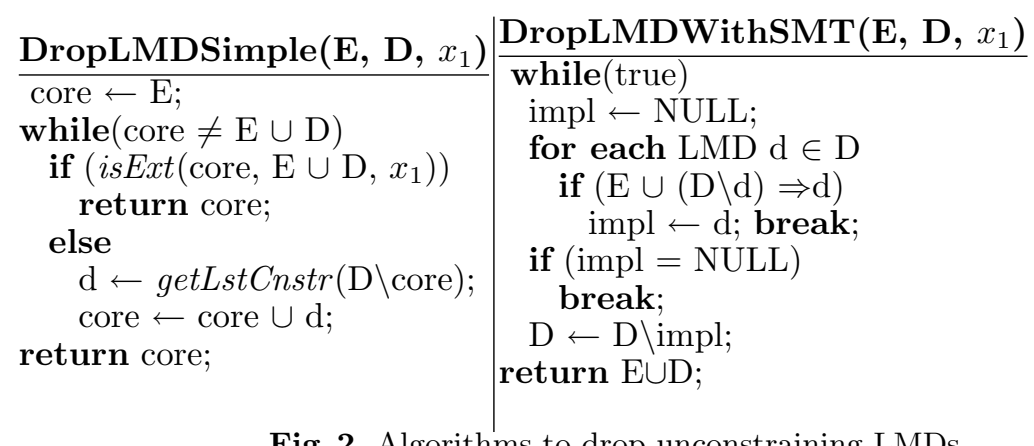

Fig. 2. Algorithms to drop unconstraining LMDs

The above idea leads to a simple algorithm, called DropLMDSimple, shown in Fig. 2. This algorithm takes as inputs a set of LMEs $E$, a set of LMDs $D$, and a variable $x_{1}$ to be quantified from the conjunction of all LMCs in $E \cup D$. Algorithm DropLMDSimple returns a subset of LMCs in $E \cup D$ such that the result of quantifying $x_{1}$ from the conjunction of LMCs in this subset is equivalent to the result of quantifying $x_{1}$ from the conjunction of LMCs in $E \cup D$.

Algorithm DropLMDSimple computes the desired subset in a variable core that is initialized to $E$. Subsequently, it determines if any solution to the conjunction of LMCs in core can be engineered by modifying specific bits of $x_{1}$ to give a solution to the conjunction of LMCs in $E \cup D$. This is achieved by invoking a function isExt. If such an engineering is indeed possible, then all LMDs not in core are unconstraining, and algorithm DropLMDSimple returns core. Otherwise we identify the LMDs in $D \backslash$ core whose truth depends on the least number of bits of $x_{1}$ using a function getLstCnstr. Intuitively, these LMDs are the most difficult ones to satisfy among the LMDs in $D \backslash$ core. These LMDs are then included in core and the process repeats. Clearly, algorithm DropLMDSimple terminates since core cannot have more LMCs than those in $E \cup D$. 
Since each LMD is of the form $2^{k_{i}} \cdot x_{1} \neq t_{i}$, the LMD with the largest $k_{i}$ is the one whose truth depends on the least number of bits of $x_{1}$. This gives a simple implementation of function getLstCnstr. One possible implementation of isExt is through the use of an SMT solver that checks if one quantified formula implies another quantified formula. However, this is inefficient in general. Instead, we propose an implementation of isExt based on the following Lemma.

Lemma 5. Let $k_{\text {core }}$ be the smallest among the $k_{i}$ 's of all LMCs in core. Let $D \backslash$ core be expressed as $\left\{\left(2^{k_{1}} \cdot x_{1} \neq t_{1}\right), \ldots,\left(2^{k_{n}} \cdot x_{1} \neq t_{n}\right)\right\}$. If $\eta=2^{k_{\text {core }}}-$ $\sum_{i=1}^{n} 2^{k_{i}} \geq 1$, any solution to the conjunction of LMCs in core can be engineered to give a solution to the conjunction of LMCs in $E \cup D$.

We give a sketch of the proof of Lemma 5 here. Let $C_{1}$ and $C_{2}$ be the conjunction of LMCs in core and the conjunction of LMDs outside core respectively. Let $\pi$ be any solution to $C_{1} . \pi$ constrains only the bits $x[0]$ through $x\left[p-k_{\text {core }}-1\right]$. Hence there are $2^{k_{\text {core }}}$ ways in which bits $x[p-1]$ through $x\left[p-k_{\text {core }}\right]$ can be assigned values such that $\pi$ remains as a solution to $C_{1}$. It can be shown that $\eta=2^{k_{\text {core }}}-\sum_{i=1}^{n} 2^{k_{i}}$ under-approximates the number of ways in which bits $x[p-1]$ through $x\left[p-k_{\text {core }}\right]$ can be assigned values such that $\pi$ becomes a solution to $C_{2}$ and remains as a solution to $C_{1}$. Therefore if $\eta \geq 1$, there exists at least one assignment of values to bits $x[p-1]$ through $x\left[p-k_{\text {core }}\right]$ such that $\pi$ becomes a solution to the conjunction of LMCs in $E \cup D$.

DropLMDSimple may not be able to identify all the unconstraining LMDs in $\exists x_{1} \cdot \psi_{2}$. For example, consider the problem $\exists x .((2 x=y) \wedge(x \neq 2 y) \wedge(x \neq$ $y)$ ), where all LMCs have modulus 8 . We have, core $=\{2 x=y\}, k_{\text {core }}=1$, $k_{1}=k_{2}=0$. Therefore, $\eta=0$ and DropLMDSimple identifies that it is not possible in general to engineer a solution of $(2 x=y)$ to give a solution of $(2 x=y) \wedge(x \neq 2 y) \wedge(x \neq y)$ by assigning values to specific bits of $x$. Hence, DropLMDSimple cannot identify any LMD to drop. However, it can be seen that $(2 x=y) \wedge(x \neq 2 y) \Rightarrow(x \neq y)$. Hence $\exists x .((2 x=y) \wedge(x \neq 2 y) \wedge(x \neq y)) \equiv$ $\exists x$. $((2 x=y) \wedge(x \neq 2 y))$. Once $x \neq y$ is dropped, DropLMDSimple can further reduce $\exists x .((2 x=y) \wedge(x \neq 2 y))$ to $\exists x$. $(2 x=y)$. Based on this idea, we present an algorithm to drop implied LMDs called DropLMDWithSMT (see Fig. 2). The notation used in this algorithm is the same as used in algorithm DropLMDSimple. The implication check in DropLMDWithSMT requires invoking an SMT solver, in general.

We now present an algorithm QE1_Layer3 which drops LMDs from $\exists x_{1} \cdot \psi_{2}$ using DropLMDSimple and DropLMDWithSMT. Given $\exists x_{1} . \psi_{2}$, QE1_Layer3 initially employs DropLMDSimple to drop unconstraining LMDs. If there still exist LMDs, DropLMDWithSMT is applied to identify the implied LMDs and drop them. If there exist LMDs in the output of DropLMDWithSMT, it is given to DropLMDSimple. Thus finally, we are left with a conjunction of LMCs $\psi_{2}^{\prime}$ with possibly fewer LMDs compared to $\psi_{2}$, while guaranteeing that $\exists x_{1} \cdot \psi_{2} \equiv \exists x_{1} \cdot \psi_{2}^{\prime}$.

The algorithms QE1_Layer1, DropLMDSimple and QE1_Layer3 form the first three layers of our layered QE algorithm. We present in Fig. 3, a proce- 
dure QE1_Layers1To3 which tries to compute $\exists x_{1} . A$ using these layers. Initially QE1_Layer 1 is called to reduce $\exists x_{1} . A$ to $\psi_{1} \wedge \exists x_{1} . \psi_{2}$. If $\psi_{2}$ is free of LMDs, QE1_1LME is called to compute $\exists x_{1} . \psi_{2}$ and hence $\exists x_{1} . A$ is computed by the first layer itself. If $\psi_{2}$ is not free of LMDs, QE1_Layers1To3 initially calls DropLMDSimple and later on QE1_Layer3 (if required) to drop the LMDs. If all the LMDs in $\exists x_{1} \cdot \psi_{2}$ are dropped by DropLMDSimple (QE1_Layer3), $\exists x_{1} . A$ gets computed in the second (third) layer. Otherwise, QE1_Layers1To3 returns $\psi_{1} \wedge \exists x_{1} . \psi_{2}^{\prime}$ such that $\psi_{1} \wedge \exists x_{1} . \psi_{2}^{\prime} \equiv \exists x_{1} . A$. The techniques to compute such (harder) instances of $\exists x_{1} . A$ are presented in the following subsection.

\subsection{Splitting and Model Enumeration}

Let us have a closer look at the instances of $\exists x_{1}$. A which cannot be computed by QE1_Layers1To3. The difficulty in QE in such cases arises from the fact that there are no LMEs constraining some of the bits of $x_{1}$ constrained by the LMDs. For example, consider the problem of computing $\exists x .((2 x=a) \wedge(x \neq b) \wedge(x \neq c))$ where all the LMCs have modulus 8 . The LME $(2 x=a)$ constrains only the bits $x[1]$ and $x[0]$ whereas the LMDs constrain the bits $x[0], x[1]$ and $x[2]$. It can be observed that in this example, QE cannot be performed by the procedure QE1_Layers1To3. We describe two techniques to compute such instances of $\exists x_{1}$. A - Splitting and Model Enumeration ${ }^{4}$.

Splitting is based on the observation that each LMD $2^{k_{i}} \cdot x_{1} \neq t_{i}$ in $A$ can be equivalently expressed as the disjunction of two constraints - an LMD $\left(2^{k} \cdot x_{1} \neq 2^{k-k_{i}} \cdot t_{i}\right)$ and a conjunction $\left(\left(2^{k} \cdot x_{1}=2^{k-k_{i}} \cdot t_{i}\right) \wedge\left(2^{k_{i}} \cdot x_{1} \neq t_{i}\right)\right)$ where $k_{i}<k$. This converts $A$ into $A_{1} \vee \ldots \vee A_{n}$ where each $A_{i}$ is a conjunction of LMCs. $\exists x_{1}$. $A$ is thus equivalent to $\exists x_{1} . A_{1} \vee \ldots \vee \exists x_{1} . A_{n}$ where each subproblem $\exists x_{1} . A_{i}$ is potentially simpler to compute than the original problem $\exists x_{1} . A$. For example, in the previous problem, the LMD $(x \neq b)$ can be split into $(2 x \neq$ $2 b) \vee((2 x=2 b) \wedge(x \neq b))$ converting the problem into $\exists x .((2 x=a) \wedge(2 x \neq$ $2 b) \wedge(x \neq c)) \vee \exists x .((2 x=a) \wedge(2 x=2 b) \wedge(x \neq b) \wedge(x \neq c))$.

Model Enumeration is based on the observation that $\exists x_{1}$. A can be equivalently expressed as $\left.\left.A\right|_{x_{1} \leftarrow 0} \vee \ldots \vee A\right|_{x_{1} \leftarrow 2^{p}-1}$ (where $\left.A\right|_{x_{1} \leftarrow i}$ denotes $A$ with $x_{1}$ replaced by constant $i$ ).

We call (i) the procedure which makes use of Splitting and Model Enumeration to compute $\exists x_{1} . A$ as QE1_Layer4 and (ii) the procedure which makes use of $Q E 1_{-}$Layer4 to compute $\exists x_{1} \cdots \exists x_{t}$. A as QE_Layer4.

We present in Fig. 3 the algorithm $Q E_{-} L M C$ which computes $\exists x_{1} \ldots \exists x_{t} . A$ using QE1_Layers1To3 and QE_Layer4. $Q E_{-} L M C$ initially tries to eliminate the quantified variables $x_{1}, \ldots, x_{t}$ one by one by applying the cheaper procedure QE1_Layers1To3. The variables which cannot be eliminated by QE1_Layers1To3 are collected in a set $Y$. It can be observed that after the for loop in $Q E_{-} L M C$, $\exists x_{1} \cdots \exists x_{t} . A$ can be equivalently expressed as $\varphi_{1} \wedge \exists Y . \varphi_{2}$ where $\varphi_{1}$ and $\varphi_{2}$ are conjunctions of LMCs (using a procedure scopeReduce in Fig. 3). $\exists Y . \varphi_{2}$ is computed by $Q E_{-} L a y e r 4$ which is conjoined with $\varphi_{1}$ to obtain the final result.

\footnotetext{
${ }^{4}$ For all the benchmarks we have experimented with, Splitting and Model Enumeration were never required to eliminate quantifiers. Hence they are only briefly described here.
} 
QE_Layer4 computes the result as a disjunction of conjunctions of LMCs. Hence the result here is, in general a Boolean combination of LMCs.

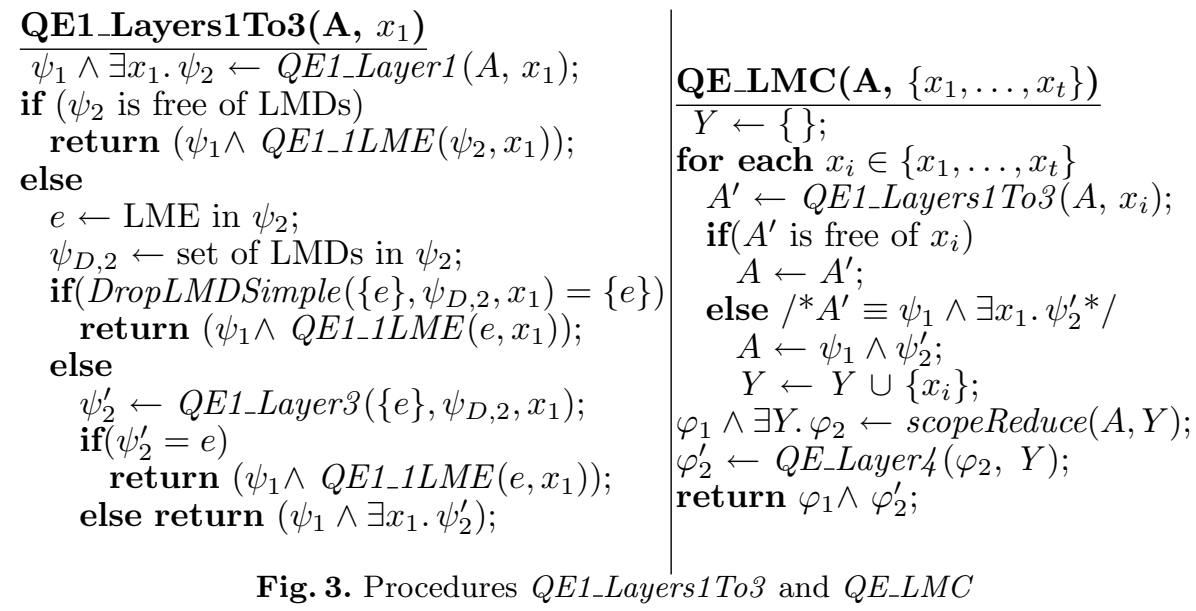

\section{Boolean Combinations of LMCs}

The QE algorithm $Q E_{-} L M C$ accepts a conjunction of LMCs. In this section, we explore two approaches for extending $Q E_{-} L M C$ to Boolean combinations of LMCs: Decision Diagram (DD) based approach and DAG based (SMT solving based) approach.

\subsection{Quantifier Elimination by DD Based Approach}

We introduce a data structure called Linear Modular Decision Diagram (LMDD) which represents Boolean combinations of LMCs. They are BDDs [4] with nodes labeled with LMEs. The problem we wish to solve in this subsection can be formally stated as follows. Given an LMDD $f$ representing a Boolean combination of LMCs over a set of variables $X$, we wish to compute an LMDD $g \equiv \exists V . f$ where $V \subseteq X$.

The algorithms presented in this subsection use the following procedures. a) createLMDD : Creates an LMDD from a DAG representing a Boolean combination of LMCs, b) isUnsat : Returns true if the conjunction of LMCs in the given set is unsatisfiable and false otherwise, d) getConjunct : Given a set of LMCs $\varphi$, returns the conjunction of LMCs in $\varphi$, e) AND, OR, NOT, ITE : Perform the basic operations on LMDDs indicated by their names. We denote a non-terminal LMDD node $f$ as $(P(f), H(f), L(f))$ where $P(f)$ is an LME, and $H(f), L(f)$ are the high child and low child respectively as defined in [4].

A straightforward procedure to compute $\exists V . f$ is to apply $Q E_{-} L M C$ to each path in $f$ similar to Black-box QE on Linear Decision Diagrams described in [1]. However, as observed in [1], this technique is not amenable to dynamic programming and the number of recursive calls to the procedure is linear in the number of paths in $f$ (which is bad).

In the following text, we present a more efficient procedure QuaLMoDE to compute $\exists V . f$. QuaLMoDE makes use of a procedure $Q E 1_{-} L M D D$ which 
eliminates a single variable $v$ from $f$ (see Fig. 4). To compute $\exists v . f$, we call $Q E 1 \_L M D D$ with arguments $f,\{\},\{\}, v . Q E 1 \_L M D D$ performs recursive traversal of $f$ carrying along each path, the set of LMEs $E$ and the set of LMDs $D$ containing $v$, encountered on the path so far (called the context). However, it tries to simplify $f$ using $E$ in the following way.

When $Q E 1_{-} L M D D$ is called with arguments $f, E, D, v$, we wish to compute $\exists v\left(f \wedge C_{E} \wedge C_{D}\right)$, where $C_{E}$ and $C_{D}$ denote the conjunctions of LMEs in $E$ and LMDs in $D$ respectively. Using Lemma 1, $E$ can be expressed as $\left\{\left(2^{k_{1}} \cdot v=t_{1}\right), \ldots,\left(2^{k_{n}} \cdot v=t_{n}\right)\right\}$. Without loss of generality, let $k_{1}$ be the minimum among $k_{1}, \ldots, k_{n}$. Let $g$ be an internal non-terminal node of $f$ denoted as $(P(g), H(g), L(g))$ with $P(g)$ expressed as $\left(2^{k} \cdot v=t\right)$ such that $k \geq k_{1}$. It can be observed that $g$ can be simplified to $\left(\left(2^{k-k_{1}} \cdot t_{1}=t\right), H(g), L(g)\right)$ using the LME $\left(2^{k_{1}} \cdot v=t_{1}\right)$. The procedures selectLME and simplifyLMDD (see Fig. 4) respectively perform the selection of LME with the minimum $k$ among the LMEs in $E$ and simplification of $f$ using the selected LME $e_{1}$ as described above. The procedure applyL1 in Fig. 4 returns an LME equivalent to the argument LME using Lemma 1.

It can be observed that simplify $L M D D$ does not require propagation of the context. If the same LMDD node is encountered with the same LME following two different paths, the results of the calls are the same. Hence simplifyLMDD can be implemented with dynamic programming.

Note that if simplify $L M D D$ is successful in eliminating all occurrences of variable $v$ using the LME selected, $Q E 1_{-} L M D D$ returns without any further recursive calls. The procedure $Q E 1_{-} L M D D$ can be repeatedly invoked to compute $\exists V$. $f$. This is implemented in the procedure QuaLMoDE.

\subsection{Quantifier Elimination by DAG Based Approach}

The problem we wish to solve in this subsection is the following. Given a DAG $f$ representing a Boolean combination of LMCs over a set of variables $X$, we wish to compute a DAG $g \equiv \exists V$. $f$ where $V \subseteq X$.

We present an algorithm Monniaux to compute $\exists V$. $f$ which is a simple extension of the algorithm EXISTELIM in [2]. EXISTELIM as given in [2] computes $\exists V$. $f$ where $f$ is a Boolean combination of linear inequalities over reals. A naive way of computing this is by converting $f$ to DNF by enumerating all satisfying assignments, and by using a QE technique for conjunctions of linear inequalities. EXISTELIM improves upon this by generalizing a satisfying assignment to obtain a cube of satisfying assignments, and by projecting the cube on the remaining variables (not in $\mathrm{V}$ ) before its complement is conjoined with $f$ and further satisfying assignments are found.

Our algorithm Monniaux is an extension of the algorithm EXISTELIM with the following changes. a) The predicates are LMCs, not linear inequalities over reals, b) the projection algorithm PROJECT [2] is replaced by $Q E_{-} L M C$, and c) the algorithm GENERALIZE2 [2] for generalization of conjunctions is replaced by an algorithm GENERALIZE2_LMC.

Given a formula $G$ and a conjunction $M$ of literals of $G$ such that $M \Rightarrow \neg G$, the algorithm GENERALIZE2 removes unnecessary literals from $M$ and returns 

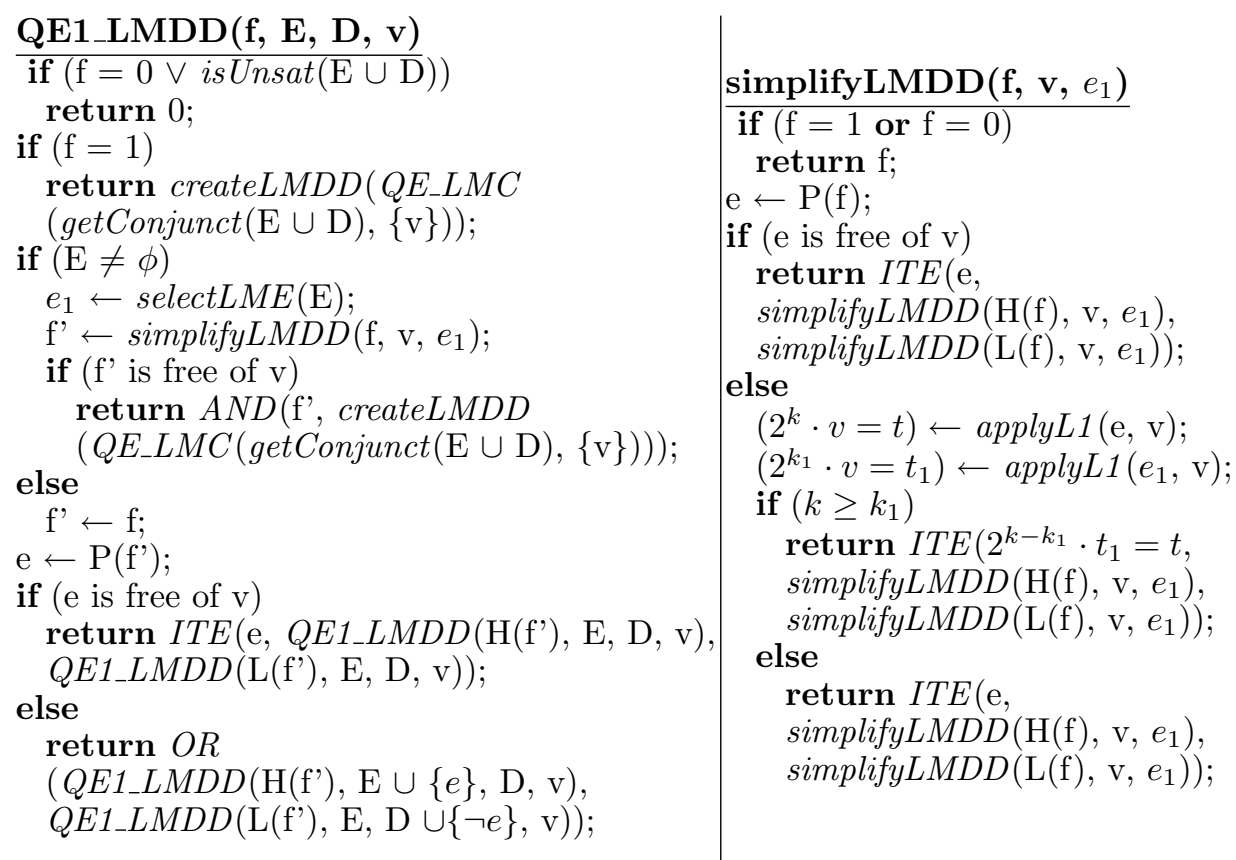

Fig. 4. Algorithms $Q E 1 \_L M D D$ and simplify $L M D D$

$M^{\prime}$ such that $M \Rightarrow M^{\prime}$ and $M^{\prime} \Rightarrow \neg G$. However, in our experiments, we have found that GENERALIZE2 is prohibitively time consuming as it involves SMT solver calls equal to the number of literals in $M$.

Our algorithm GENERALIZE2_LMC works in the following way. Note that $M$ assigns a Boolean value to each atomic predicate of the formula $\neg G$. We evaluate the propositional skeleton (DAG representation of the propositional structure) $P$ of $\neg G$ using these Boolean values assigned to the atomic predicates. This assigns a Boolean value $b_{n}$ to each node $n$ in $P$. We now find the subset $S_{n}$ of literals in $M$ which is sufficient to evaluate $n$ to $b_{n}$. Let $S_{r}$ be the set of literals found in this way for the root $r$ of $P$. Let $M^{\prime}$ be the conjunction of literals in $S_{r}$. It is easy to see that $M \Rightarrow M^{\prime}$ and $M^{\prime} \Rightarrow \neg G$. We illustrate this idea with the help of an example. Let $\neg G$ be the formula ite $(A, B, C) \vee$ ite $(D, E, F)$ and let $M$ be $A \wedge B \wedge \neg C \wedge \neg D \wedge \neg E \wedge F$ where $A, B, C, D, E$ and $F$ are LMCs. It is easy to see that the set of literals $\{A, B\}$ is sufficient to evaluate ite $(A, B, C)$ to true. Similarly $\{\neg D, F\}$ is sufficient to evaluate ite $(D, E, F)$ to true. Hence, it follows that $\{A, B\}$ (or $\{\neg D, F\}$ ) is sufficient to evaluate $\neg G$ to true. Hence GENERALIZE2_LMC returns $A \wedge B$ (or $\neg D \wedge F)$ as $M^{\prime}$.

\section{Experimental Results}

We conducted three sets of experiments a) to evaluate QuaLMoDE, Monniaux and $Q E_{-} L M C$, b) to compare the performance of $Q E_{-} L M C$ with alternative QE techniques and c) to evaluate the utility of our $\mathrm{QE}$ algorithms in verification.

The experiments are performed on a $1.83 \mathrm{GHz}$ Intel(R) Core 2 Duo machine with 2 GB memory running Ubuntu 8.04. We have implemented our own LMDD 
package for carrying out the QE experiments by DD based approach. In our implementation, we convert LMDs with modulus 2 to equivalent LMEs as a simplification step. Hence, in this section "LMD" refers to LMD with modulus greater than 2 .

Evaluation of QuaLMoDE, Monniaux and $Q E_{-} L M C$ : In order to evaluate QuaLMoDE and Monniaux, we used a benchmark suite consisting of 210 real benchmarks and 212 artificial benchmarks. The real benchmarks are derived from real word-level VHDL designs. We created these benchmarks by obtaining the transition relations $(R)$ of these designs and then (i) computing abstract transition relation by quantifying out the internal variables of the design from $R$, (ii) computing the set of states reachable in $2^{i}$ steps using iterative squaring.

We observed a significant number of LMDs in these benchmarks when expressed in Negation Normal Form (NNF) (see Fig. 5(a)). In order to generate the artificial benchmarks, we selected some of the real benchmarks and some SMTLib benchmarks from the category QF_BV/bruttomesso/simple_processor/ of the SMTLib fixed size bit-vector benchmarks [10] and used different random choices for the set of variables to be eliminated ${ }^{5}$. The number of variables $(N)$, number of variables to be eliminated $(E)$ and the number of bits to be eliminated in the benchmarks range from 3 to 175,1 to 170 and 1 to 1265 respectively.
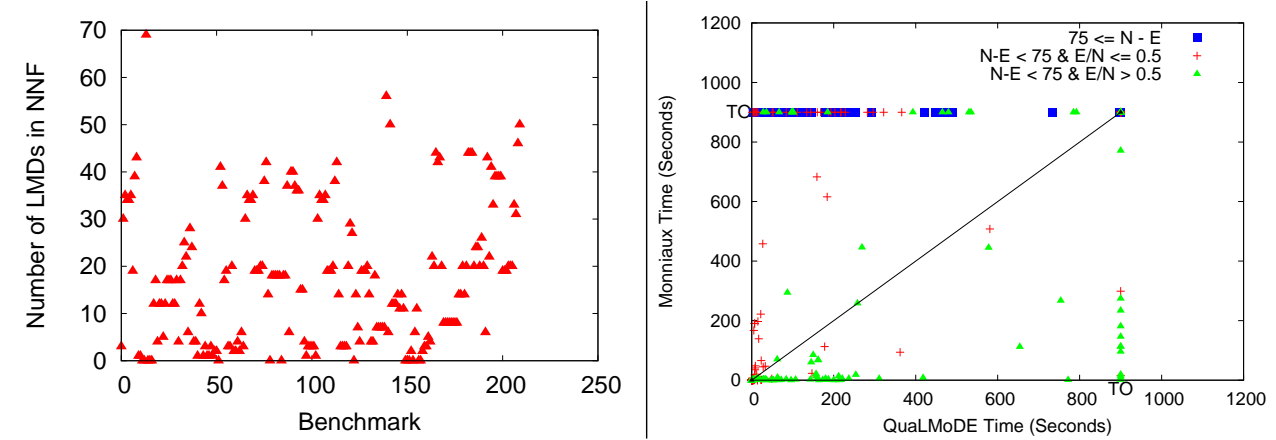

Fig. 5. Plots showing (a) significant number of LMDs in the real benchmarks. (b) QuaLMoDE Time Vs Monniaux Time (TO : > 900 seconds)

We measured the QE time by QuaLMoDE and Monniaux for each benchmark (For QuaLMoDE, this includes the time taken to build the LMDD also). It is observed that (see Fig. 5(b)), for the benchmarks with $N-E$ below a certain threshold $t_{1}$ and $E / N$ above a certain threshold $t_{2}$, Monniaux performs better than $Q u a L M o D E$ in most of the cases (For our benchmark suite, $t_{1}$ and $t_{2}$ were empirically estimated as 75 and 0.5 respectively). For the other benchmarks, QuaLMoDE outperforms Monniaux. It is also observed that, for the benchmarks with $t_{1} \leq N-E$, Monniaux times out irrespective of $E / N$. We figured out that this is due to the following reasons. (i) For the benchmarks with low $N-E$ and high $E / N$, the interleaving of projection inside model enumeration

\footnotetext{
5 The SMTLib benchmarks contain bit-vector operators like selection and concatenation which our work does not address. We introduced a fresh variable to denote the result of each such operator.
} 

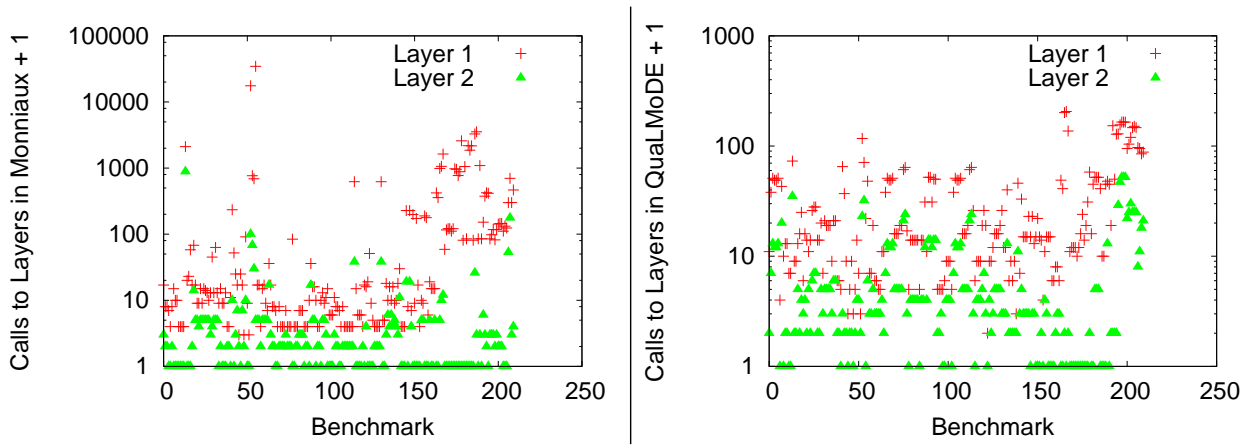

Fig. 6. Contribution of the layers in $Q E_{-} L M C$

in Monniaux simplifies the problem considerably whereas for the other benchmarks this simplification is not substantial. (ii) The single variable elimination strategy in $Q u a L M o D E$ results in more calls to $Q E 1_{-} L M D D$ from $Q u a L M o D E$ for benchmarks with low $N-E$ and high $E / N$.

The number of calls to QE_LMC from QuaLMoDE and Monniaux while performing QE from the real benchmarks ranges from 1 to 205 and 1 to 3842 respectively. We observed that a considerable number of these calls contain LMDs. The average number of LMDs in these $Q E_{-} L M C$ calls from QuaLMoDE and Monniaux ranges from 0 to 12.2 and 0 to 18.8 respectively and the average of the ratio of the number of LMEs to the number of LMDs ranges from 0 to 1 and 0.19 to 23.4 respectively.

We evaluated the contribution of different layers of $Q E_{-} L M C$ in performing $\mathrm{QE}$ from the real benchmarks. It was observed that all the quantifiers were eliminated by the first two layers - without even a single call to QE1_Layer3 or QE_Layer4. A large fraction of the calls to QE1_Layers1To3 were solved by the first layer itself and the remaining by the second layer (see Fig. 6$)^{6}$.
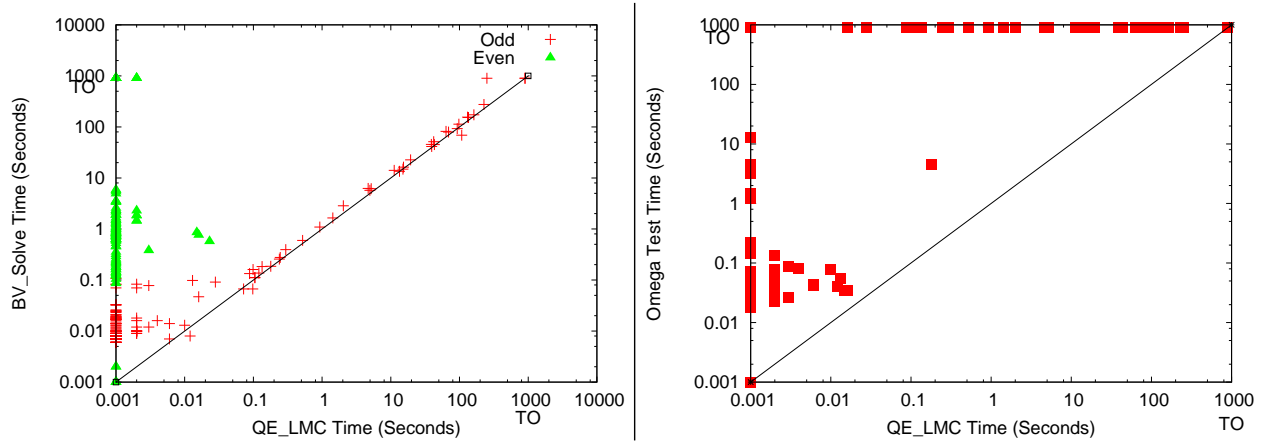

Fig. 7. Plots comparing (a) $Q E_{-} L M C$ with $B V_{-} S o l v e$ (b) $Q E_{-} L M C$ with Omega Test (TO : > 900 seconds)

Comparison of $Q E_{-} L M C$ with alternative $Q E$ techniques : We compared the performance of $Q E_{-} L M C$ with QE based on Presburger Arithmetic using Omega Test and QE based on bit-blasting (see Fig. 7). In the latter case, we have used a procedure $B V_{-}$Solve which performs the elimination of quanti-

\footnotetext{
${ }^{6}$ Note that the y-axis of both the plots are in log-scale. One is added to the y-values to include the points with no calls to the second layer.
} 
fied variables appearing with odd coefficients in LMEs using the ideas described in [6] followed by bit-blasting and bit-level QE using [11]. We used a set of 405 benchmarks which are instances of the $\mathrm{QE}$ problem at conjunction level 371 of them arise from QuaLMoDE/Monniaux when QE is performed on the real benchmarks and 34 are randomly generated. Our results clearly demonstrate that $Q E_{-} L M C$ outperforms these alternative QE techniques. In Fig. 7(a), a benchmark is labeled "Odd" if each quantified variable in it appears with odd coefficient in at least one LME and "Even" otherwise. Our results demonstrate that BV_Solve performs comparable to $Q E_{-} L M C$ for the "Odd" benchmarks, but not for the "Even" ones. This is not surprising; since $B V_{-}$Solve uses the technique from [6] to eliminate variables whenever possible before bit-blasting, it is able to eliminate variables without any bit-blasting for all "Odd" benchmarks. In contrast, $B V_{-}$Solve has to bit-blast for "Even" benchmarks, thereby performing poorly.

Utility of our QE algorithms in verification : In order to evaluate the utility of our QE algorithms, we used QuaLMoDE to compute abstract transition relations when doing BMC of word-level VHDL designs. We derive the transition relation $R$ of the design and then for each BMC frame $i$, we obtain a slice $R_{i}$ of $R$ containing only relevant part of $R$ for this frame. Next we eliminate a chosen subset of variables (subset of internal variables) from $R_{i}$ to obtain $R_{i}^{\prime}$ using QuaLMoDE as well as $Q B V_{-}$Solve (an extension of $B V_{-}$Solve using the DD based approach). The final unrolled constraint is a conjunction of the different $R_{i}^{\prime}$ s computed by $Q u a L M o D E / Q B V_{-}$Solve which after conjoining with the negation of the property is given to an SMT solver for proving/refuting. The SMT solver used is simplifyingSTP $[12]^{7}$. Table 1 gives a summary of these results. The designs machine_1 to machine_12 are modified versions of publicly available benchmarks obtained from [9]. The remaining designs are proprietary and were obtained from safety critical applications used in nuclear reactors. They are control-oriented designs with wide data paths. Our results clearly demonstrate (i) the significant performance benefit of using abstract transition relations computed by $Q u a L M o D E$ in these verification exercises and (ii) the performance upper hand of QuaLMoDE over $Q B V_{-}$Solve in computing the abstract transition relations particularly for designs involving constant multiplications with even coefficients and large bit widths.

Our QE algorithms can be used for solving Boolean combinations of LMCs by quantifying out all the variables. However our preliminary experiments suggest that this approach is not competitive with DPLL-style SMT solvers or bit-blasting followed by QBF solving.

\section{Conclusion}

In this paper, we tackled the QE problem for LMCs. Our main contributions are : (i) A bit-blasting-free QE algorithm for conjunctions of LMCs which is later extended to QE algorithm for Boolean combination of LMCs, (ii) comparison of

\footnotetext{
${ }^{7}$ We selected simplifyingSTP because (i) it is the winner of SMT-COMP 2010 bitvector category and (ii) it has a variable eliminator implemented as per [6].
} 
Table 1. Experimental Results on VHDL Programs

\begin{tabular}{|c|c|c|c|c|c|c|}
\hline \multirow{2}{*}{ Design } & \multirow{2}{*}{ LOC } & \multirow{2}{*}{ SS } & \multirow{2}{*}{ TR } & \multicolumn{3}{|c|}{$\mathrm{UNR}=\mathbf{5 0 0}$} \\
\hline & & & & NA & QL & QB \\
\hline machine_1 & 363 & 8 & $(371,20,547)$ & $\overline{\mathrm{TO}(\mathrm{TO})}$ & $98(4,27)$ & $\overline{\mathrm{TO}(\mathrm{TO},-)}$ \\
\hline machine_2 & 373 & 6 & $(371,19,341)$ & TO(TO) & $70(2,0)$ & $\mathrm{TO}(\mathrm{TO},-)$ \\
\hline machine_3 & 383 & 7 & $(395,22,344)$ & $\mathrm{TO}(\mathrm{TO})$ & $75(3,3)$ & $\mathrm{TO}(\mathrm{TO},-)$ \\
\hline machine_4 & 253 & 4 & $(235,19,515)$ & $1497(1418)$ & $79(1,0)$ & $\mathrm{TO}(\mathrm{TO},-)$ \\
\hline machine_5 & 253 & 4 & $(235,19,387)$ & $1527(1451)$ & $76(1,0)$ & $\mathrm{TO}(\mathrm{TO},-)$ \\
\hline machine_6 & 363 & 4 & $(242,15,56)$ & $122(80)$ & $41(0,0)$ & $52(2,3)$ \\
\hline machine_7 & 379 & 5 & $(270,20,61)$ & $206(152)$ & $52(3,1)$ & $66(3,5)$ \\
\hline machine_8 & 251 & 2 & $(170,13,83)$ & $225(195)$ & $30(1,1)$ & $35(4,1)$ \\
\hline machine_9 & 251 & 3 & $(170,13,323)$ & $\mathrm{TO}(\mathrm{TO})$ & $30(1,1)$ & $53(28,1)$ \\
\hline machine_10 & 363 & 5 & $(242,15,356)$ & $\mathrm{TO}(\mathrm{TO})$ & $40(1,0)$ & $63(13,3)$ \\
\hline machine_11 & 363 & 6 & $(352,22,96)$ & TO(TO) & $97(1,7)$ & $98(2,24)$ \\
\hline machine_12 & 363 & 5 & $(242,15,356)$ & $\mathrm{TO}(\mathrm{TO})$ & $478(8,427)$ & $\mathrm{TO}(\mathrm{TO},-)$ \\
\hline board_1 & 404 & 4 & $(265,13,163)$ & $1455(1426)$ & $51(24,0)$ & $\mathrm{TO}(\mathrm{TO},-)$ \\
\hline board_2 & 373 & 3 & $(283,13,163)$ & $\mathrm{TO}(\mathrm{TO})$ & $66(49,0)$ & $\mathrm{TO}(\mathrm{TO},-)$ \\
\hline board_3 & 503 & 4 & $(284,13,190)$ & $\mathrm{TO}(\mathrm{TO})$ & $67(44,0)$ & $\mathrm{TO}(\mathrm{TO},-)$ \\
\hline board_4 & 415 & 3 & $(272,11,31)$ & $362(229)$ & $111(10,3)$ & $215(104,13)$ \\
\hline
\end{tabular}

All times are in seconds. TO : > 1800 seconds, LOC : Lines of code, SS : Symbolic simulation

time, TR : Transition relation details (dag size, number of variables, number of bits), NA : Without abstraction : total time (simplifyingSTP time), QL : With QuaLMoDE for abstraction : total time ( $Q u a L M o D E$ time, simplifyingSTP time), QB : With $Q B V \_$Solve for abstraction : total time ( $Q B V_{-}$Solve time, simplifyingSTP time) (for NA, QL and QB most of the remaining time is spent in slicing - we use a naive implementation of slicer), UNR : Number of BMC unrollings

our approach with alternative techniques and the identification of a simple-touse criteria for choosing the right QE approach for a given problem instance. We propose to study QE from linear modular inequalities as part of future work.

Acknowledgements : We would like to thank Trevor Hansen and Vijay Ganesh for providing us with the latest version of simplifyingSTP. We also thank Mukesh Sharma, Ashutosh Kulkarni, Rajkumar Gajavelly and Nachiket Vaidya for their valuable support. We convey our special acknowledgement to Anup Bhattacharjee and S.D.Dhodapkar for their indispensable help and support.

\section{References}

1. S. Chaki, A. Gurfinkel, O. Strichman. Decision diagrams for linear arithmetic, In FMCAD 2009

2. D. Monniaux. A quantifier elimination algorithm for linear real arithmetic, In LPAR 2008

3. D. Kroening, O. Strichman. Decision procedures : an algorithmic point of view, Texts In Theoretical Computer Science, Springer 2008

4. R.E. Bryant. Graph-based algorithms for boolean function manipulation. IEEE Transactions on Computers, C-35(8):677-691, 1986

5. W. Pugh. The Omega Test : a fast and practical integer programming algorithm for dependence analysis. Communications of the ACM, Pages 102-114, 1992

6. V. Ganesh, D. Dill. A decision procedure for bit-vectors and arrays, In CAV 2007

7. H. Jain, E. M. Clarke, O. Grumberg. Efficient Craig interpolation for linear diophantine (dis)equations and linear modular equations, In CAV 2008

8. V. Ganesh, S. Berezin, D. Dill. Deciding Presburger arithmetic by model checking and comparisons with other methods, In FMCAD 2002

9. ITC'99 benchmarks, http://www.cad.polito.it/downloads/tools/itc99.html

10. SMTLib website, http://goedel.cs.uiowa.edu/smtlib/

11. CUDD release 2.4 .2 website, vlsi.colorado.edu/ fabio/CUDD

12. STP website, http://sites.google.com/site/stpfastprover/

13. http://www.cse.iitb.ac.in/ supratik/software/qualmode 